\title{
Results of treatment of osteoarthritis of the scaphotrapeziotrapezoidal joint with tendon allograft interposition
}

\author{
Annelies Eeckhoudt, Nadine Hollevoet \\ From the Department of Orthopaedic Surgery and Traumatology University Hopsital Ghent
}

The aim of the study was to determine if the use of tendon allografts in combination with distal scaphoid resection for the treatment of isolated STT arthrosis is a save procedure. We reviewed the postoperative complications, re-operations, clinical and radiological results of this treatment modality. A retrospective cohort study was conducted. Investigated parameters include wrist mobility (wrist extension and -flexion), strength (grip- and pinch strength), patient-reported outcome scores : Visual Analogue Scale (VAS), Quick Disabilities of the Arm, Shoulder and Hand score (Q-DASH) and Patient Rated Wrist/Hand Evaluation score (PRWHE) and radiographic measurements : scapholunate (SL) angle, radiolunate (RL) angle and capitolunate (CL) angle. Ten wrists were included in nine patients. No revision surgery was performed. Two patients had transient neuropraxia of the radial nerve. Postoperative flexion-extension arc was $112^{\circ}$. Grip-strength was significantly increased after surgery (20 to $28 \mathrm{~kg})$. The average VAS score the past week was 1.75 (range 0-6.7), the average maximum VAS score was 3.0 (range 0-10). The mean PRWHE score was 16.6 (range 0- 69). The mean Q-DASH score was 17.95 (range 0-51). The current study indicates that distal scaphoid resection for isolated STT arthritis is a save procedure with minimal complications. It significantly improves grip strength. Mobility of the wrist was similar to contralateral wrist after surgery. Pain postoperatively was very limited (low VAS scores) and good functional scores (Q-DASH and PRWHE) were noted. Our findings support the prior findings that excisional arthroplasty might worsen carpal instability.

No benefits or funds were received in support of this study. None of the authors have a conflict of interest.
Keywords : scaphotrapeziotrapezoidal osteoarthritis ; scaphoidectomy ; tendon allograft ; carpal instability.

\section{INTRODUCTION}

Osteoarthritis off the scaphotrapeziotrapezoidal (STT) joint is the second most common site of radiographic osteoarthritis in the wrist after thumb trapezial-metacarpal joint osteoarthritis (1). Symptomatic isolated STT joint arthritis can affect up to $16 \%$ of the population (2).

Clinical signs include radial-sided wrist pain, swelling, tenderness over the STT joint (3) and pain during activities that require a large range of wrist motion (4). On clinical examination STT arthrosis presents with more medial and proximal pain compared to thumb trapezial-metacarpal joint symptoms (3).

Annelies Eeckhoudt,

ENadine Hollevoet

Department of Orthopaedic Surgery and Traumatology University Hopsital Ghent, Belgium.

Correspondence : Eeckhoudt Annelies, Department of Orthopedic Surgery, Ghent University Hospital, C. Heymanslaan 10, 9000 Gent, Belgium. Tel. : +32 4988002 63, Fax : +32 93324975 .

Email : annelies.eeckhoudt@ugent.be

- 2021, Acta Orthopædica Belgica. 
Initial treatment of STT arthritis should be conservative and involves splinting, activity modification, anti-inflammatory medication and steroid injections for pain relief (3). Painful STT arthritis tends to respond well to conservative treatment (5).

The literature suggests a wide range of possible surgical techniques for management of isolated STT osteoarthritis. This reflects the lack of consensus to the most effective treatment (6). Surgical procedures for symptomatic STT arthrosis include: distal scaphoid excision, with or without filling of the void (with autologous tendon graft or pyrocarbon implant), trapeziectomy with or without partial trapezoidectomy, STT arthrodesis, arthroscopic debridement or distal scaphoid excision (3), wrist denervation (7) or resurfacing implant of the distal pole of the scaphoid (INCA prosthesis, Arseus Medical group).

Advantages of the STT resection arthroplasty include a technically easier operation, shorter immobilization period, faster recovery for the patient, and less complications compared to arthrodesis (4). One of the main concerns on the treatment of STT resection arthroplasty is the development or worsening of midcarpal instability $(8,9)$. Shortening of the scaphoid decreases the flexion moment of the scaphoid and allows the extension moment of the lunate to predominate. This leads to an extension of the proximal row and eventually can lead to dorsal intercalated segment instability (DISI) deformity (9).

The aim of the study was to determine if the use of tendon allografts in combination with distal scaphoid resection is a save procedure. The second goal was to investigate the effect of surgery on functional and patient reported outcome measures. Third we examined the effect of amount of resected distal scaphoid and the change in radiographic parameters on functional- and patient reported outcome measures.

\section{MATERIALS AND METHODS}

We conducted a retrospective cohort study of patients who were treated with an open distal pole resection of the scaphoid with tendon allograft interposition for isolated STT arthritis between
2006 and 2020. A total of 10 treated wrists were identified, in nine patients. There were no patients lost to follow-up. All participants gave written informed consent. The authors obtained approval by the institutional ethical committee (BC-07332). All operations were performed by a senior hand surgeon (NH). The follow-up examination and was done by the first author (AE). Surgery was performed under general anaesthesia, an upper arm tourniquet was used. The STT joint was first located under fluoroscopy. A dorso-radial incision was made over the STT joint. Further dissection between the tendons of the first extensor compartment was performed down to the joint capsule. The STT capsule was longitudinally opened dorsally. A minimal resection of the distal scaphoid was performed. The plane of the scaphoid osteotomy was parallel to the proximal trapeziotrapezoid articular surface. A donor tendon was rolled-up like an anchovy and sutured together with ethybond 2.0 suture, and placed in the empty space. The dorsal capsule was reattached. Postoperative the wrist was immobilised during 4 weeks in a forearm cast. Afterwards a brace was applied and patients were instructed to mobilise the wrist and thumb.

We noted demographic parameters (age at time of surgery, gender, side of surgery, dominance), postoperative complications and revision surgery. Patient reported outcome, functional outcome measurements and radiographs (posteroanterior and lateral view) of both wrists and were obtained. Patient-reported outcomes were evaluated using a Visual Analogue Scale (VAS), Quick Disabilities of the Arm, Shoulder and Hand score Dutch Language Version (Q-DASH-DLV) (10) and Patient Rated Wrist/Hand Evaluation score Dutch Language Version (PRWHE-DLV) (11). VAS score defines a score from 0 to 10 ( 0 no pain to 10 worst possible pain). Patients had to answer two questions concerning their pain perception: 'How high would you rate the average pain of the past 7 days?' (= average VAS score the past week) and 'How high would you rate the most intense pain you perceived the last 7 days?' (=maximum VAS score). The Q-DASH is a shortened version of the DASH questionnaire, which is a scoring system for disability of the upper extremity. The questionnaire 


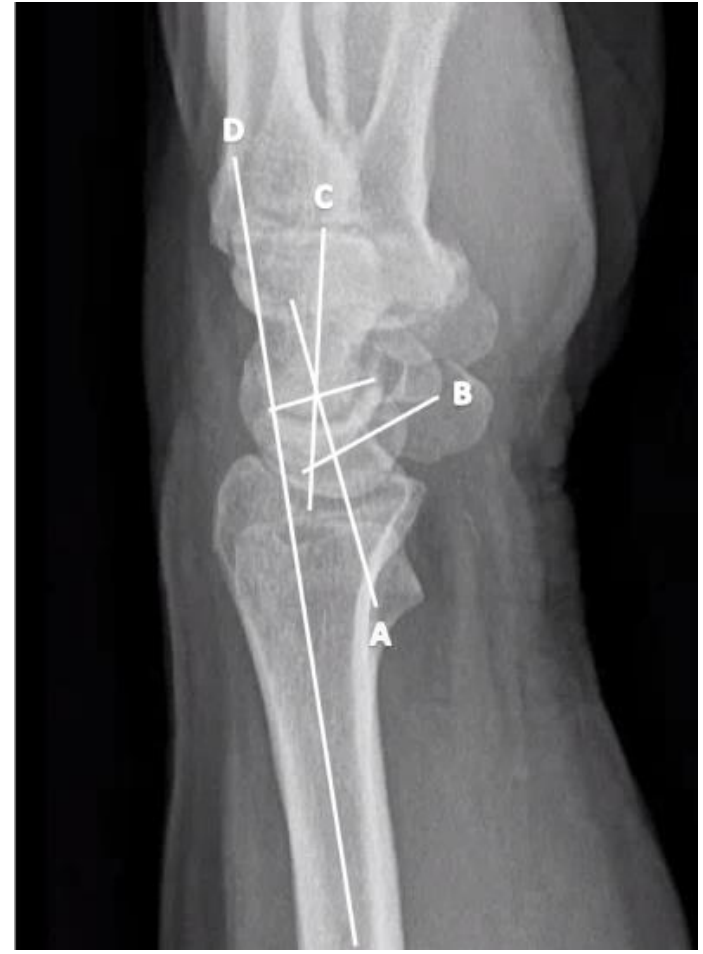

Figure 1. - Radiographic axes used for radiographic measurements. A : lunate axis, defined as the line perpendicular to perpendicular to the line connecting the palmar and dorsal horns of the lunate, $\mathrm{B}$ : scaphoid axis defined as a line connecting the midpoint of its proximal and distal pole, $\mathrm{C}$ : capitate axis, line through midportion of its proximal pole to the midportion of its distal articular surface. D : Axis of the radius, defined by the line passing the middle of the sagittal diameter of the radius.

contains 2 optional modules on work and instrument and sports, that were not included. The scores range from 0 (no disability) to 100 (complete disability). The PRWHE is a questionnaire containing 15 questions concerning pain and functionality (specific and daily functioning). Each question is rated from 0 to 10 ( 0 no pain/no difficulty to 10 worst possible pain/ not capable of performing). This provides a total score of 0 to 100. A higher score is associated with more pain and disability and thus with a poorer outcome. Functional outcome measurements included range of motion of the wrist, grip strength, and key pinch. Active range of motion of the wrist was measured in extension and flexion using a goniometer. Thumb opposition was assessed according to Kapandji's classification (12). Grip strength was measured in kilograms using a
Jamar hand dynamometer. Key pinch was measured in kilograms using a hydraulic pinch gauge. For each outcome, the average of 3 repeated measurements was obtained. Bilateral radiographs were taken in posteroanterior and lateral view; we measured the scapholunate (SL) angle, capitolunate angle (CL) and radiolunate (RL) angle. Radiographic parameters were measured based on radiographic axes as illustrated in figure 1 (13). In accordance with several authors, the following normal range of radiographic values were considered : SL angle of $30^{\circ}-60^{\circ}$, RL angle of $-15^{\circ}$ to $15^{\circ}$ and $\mathrm{CL}$ angle of $-15^{\circ}$ to $15^{\circ}$ (13). The degree of STT osteoarthritis was determined on preoperative radiographs according to White et al. (14). Resection of distal scaphoid was measured in $\mathrm{mm}$ on postoperative frontal radiograph of the wrist and was calculated by the difference of the postoperative length of the scaphoid to the preoperative length.

Statistical analysis was performed with MedCalc (MedCalc Software, Ostend, Belgium) and SAS (SAS Institute, NC, USA). To compare preoperative measurements (grip strength and radiographic parameters such as SL angle, RL angle and CL angle) with postoperative measurements the Student $\mathrm{T}$ test was used, and the level of significance was set at 0.05 . The difference in mobility (wrist flexion and extension) and grip -and pinch strength for the operated and contralateral side, was analysed using the student $\mathrm{T}$ test, significance level was set at 0.05 . To evaluate the effect of amount of resected distal scaphoid on wrist mobility, strength, pain and patient reported outcome score the Pearson correlation analysis $(\mathrm{R}=$ Pearson correlation coefficient) was used.

\section{RESULTS}

Ten wrist were included, in 9 patients (one patient was operated on both sides). Six women were operated and 4 men. Mean age at time of operation was 57 years (range 37-74 year). Time to radiographic follow up was 61.6 months (range 5-166 months) and mean time to functional scoring and patient-reported outcome follow-up was 92.9 months (range 11-166 months). Three patients were not able to visit the hospital because of the COVID 


\begin{tabular}{|c|c|c|c|c|c|c|c|c|c|c|c|}
\hline & ННМУd & a & 8 & $=$ & $-c$ & 0.4 & $n$ & & $\infty$ & $\underline{2}$ & 官 \\
\hline & HSVA - ठ & $\stackrel{n}{i}$ & $\vec{n}$ & 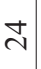 & 0 & 0.4 & $n$ & & $\infty$ & $m$ & 管 \\
\hline \multicolumn{2}{|c|}{ 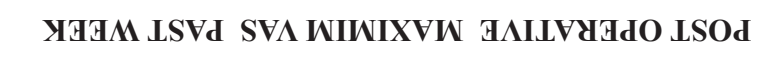 } & - & $\circ$ & - & 0 & or & $\sqrt{0}$ & $\theta$ & 0 & $\sim$ & $\vec{m}$ \\
\hline \multicolumn{2}{|c|}{ 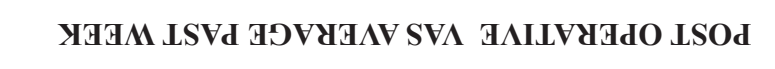 } & $\because$ & $\stackrel{\sim}{+}$ & - & 0 & 0 is & r & & 0 & 0 & $\stackrel{?}{\stackrel{2}{=}}$ \\
\hline \multirow{2}{*}{ 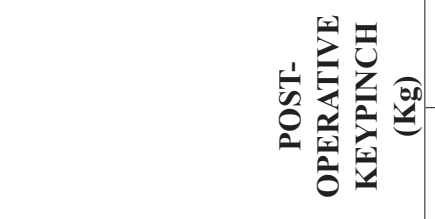 } & 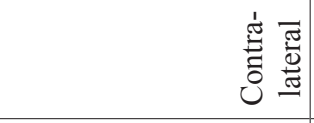 & $n$ & $\wedge$ & $\because$ & $a-$ & +4 & in 0 & P & $n$ & & $\bullet$ \\
\hline & 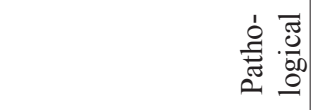 & $\stackrel{?}{+}$ & $\because$ & in & $\simeq 4$ & $n=$ & $+\sin$ & & 0 & & 6 \\
\hline \multirow{2}{*}{ 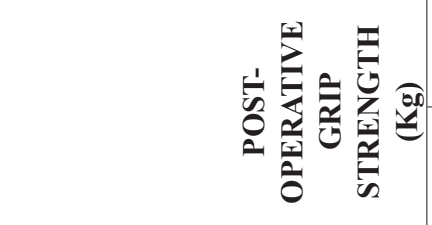 } & 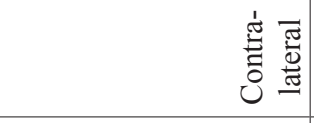 & \& & $\approx$ & $\vec{n}$ & $\ddot{m}:$ & \pm 0 & $\sim{ }_{\mu}^{\infty}$ & & $\approx$ & i & e् \\
\hline & 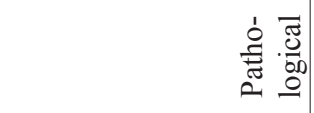 & $\approx$ & $\hat{\imath}$ & in & J & $\vec{\sim}$ & $E \propto$ & & $\Xi$ & $\stackrel{\sim}{\sim}$ & $\stackrel{\infty}{\sim}$ \\
\hline \multirow{2}{*}{ 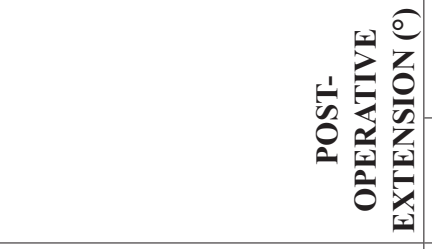 } & 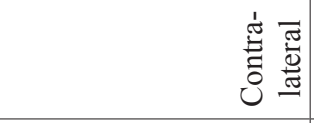 & f & 8 & 8 & 85 & 83 & $\widetilde{\delta} \varsubsetneqq$ & & \& & & in \\
\hline & 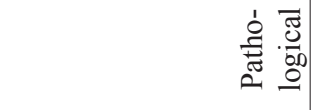 & 8 & ชె & $尺$ & 85 & 8 & 87 & & i & 8 & i \\
\hline \multirow{2}{*}{ 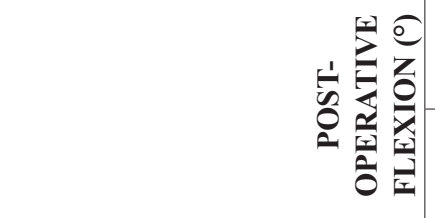 } & 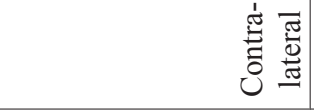 & 8 & ¿ & ช్ & $i n$ & 83 & $\widetilde{\delta} / \underset{\sim}{\infty}$ & & $i$ & & is \\
\hline & 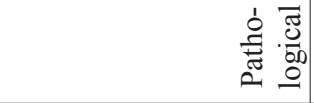 & 8 & 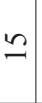 & $\infty$ & $R s$ & તָ) & $8 \%$ & & $\stackrel{i}{n}$ & 8 & in \\
\hline \multicolumn{2}{|c|}{ (шس) NOILOASAY HO LNOONV } & in & $m$ & $N$ & $N$ & 0 & +0 & $\nabla$ & $m$ & in & $\stackrel{m}{+}$ \\
\hline \multicolumn{2}{|c|}{ 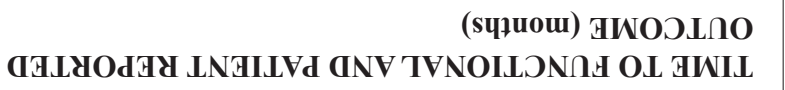 } & $=$ & $m$ & $\stackrel{m}{=}$ & 8 & 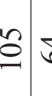 & $\Xi \nsubseteq$ & $\stackrel{n}{m}$ & $\stackrel{\circ}{2}$ & $\tilde{n}$ & $\dot{d}$ \\
\hline \multicolumn{2}{|c|}{ (sч) } & $=$ & $m$ & $\stackrel{\overbrace{}}{\varrho}$ & $\stackrel{y}{0}$ & 6 & tg & $\bar{\lambda}$ & $\infty$ & $n$ & 官 \\
\hline \multicolumn{2}{|r|}{ GPNVNIWOA } & $\stackrel{\circ}{z}$ & $\stackrel{\circ}{z}$ & $\stackrel{\circ}{z}$ & 20 & 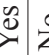 & 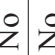 & 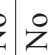 & $\stackrel{8}{z}$ & $\overbrace{}^{\infty}$ & \\
\hline \multicolumn{2}{|r|}{ GAIS AG.LVYGAO } & 离 & 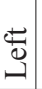 & 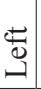 & $\vec{t}: \overrightarrow{0}$ & to. & $\frac{5}{9} \cdot \frac{1}{6}$ & & 穿 & $\frac{\vec{t}}{.00}$ & \\
\hline \multicolumn{2}{|r|}{ унवNसФ } & 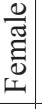 & $\sum^{\frac{0}{\pi}}$ & $\frac{0}{\pi}$ & $\frac{0}{\pi}$ & $\begin{array}{c}0 \\
0 \\
0 \\
0 \\
\vdots \\
5\end{array}$ & 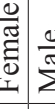 & & 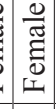 & 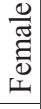 & \\
\hline \multicolumn{2}{|r|}{ HOV } & t & in & $\therefore$ & $\tilde{n}$ & in & $n 8$ & in & $\frac{1}{4}$ & $\hat{m}$ & in \\
\hline & & 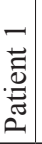 & 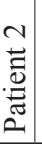 & 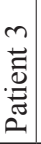 & 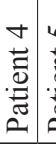 & 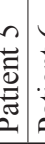 & 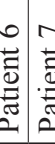 & & 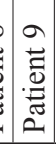 & & $\frac{z}{2}$ \\
\hline
\end{tabular}


pandemic. For these patients data was extracted from their patient files and questionnaires were obtained electronically. Seven patients were operated on the non-dominant hand. STT arthrosis was classified using the White classification (14): two patients were classified in stage 1 , three in stage 2 and four in stage 3 . None of the patients had revision surgery. Two patients had a transient neuropraxia of the radial nerve. Summary of postoperative mobility, strength and patient-reported outcome measures were presented in Table I. Mobility postoperatively did not change significant compared to contralateral wrist (wrist flexion $\mathrm{p}=0.72$, wrist extension $\mathrm{p}=0.08$ ). Postoperative Kapandji score was $10 / 10$ for all patients. Postoperative strength significantly increased from $20 \mathrm{~kg}$ preoperative to $28 \mathrm{~kg}$ postoperative $(\mathrm{p}=0.002)$. Grip strength postoperative was $95 \%$ of the contralateral side. Keypinch postoperative was similar for both sides (both sides 6kg).

The average VAS score the past week was 1.75 (range 0-7), the average maximum VAS score was 3.0 (range 0-10). The mean PRWHE score was 16.6 (range 0-69). The mean Q-DASH score was 17.95 (range 0-51). Radiographic parameters that may influence postoperative carpal instability were summarized in Table II. Five out of ten operated wrists had a SL angle $>60^{\circ}$ after surgery, in three wrists the CL angle was $>15^{\circ}$ and in three wrists the RL angle was $>15^{\circ}$ after surgery. A mean resection of the distal scaphoid of $4.3 \mathrm{~mm}$ was measured (range $2.0 \mathrm{~mm}-9.0 \mathrm{~mm}$ ). No correlation was found between amount of resection or radiographic parameters and patient reported outcome measures (Q-DASH and PRWHE).

\section{DISCUSSION}

We compared the postoperative results of different treatment modalities for STT arthritis to the results of the current study. Flexion-extension arc in the present study of $113^{\circ}$ was comparable to $118^{\circ}$ reported by Garcia-Elias (15) and $135^{\circ}$ by Berkhout etal (16) who performed a distal scaphoid resection with or without tendon interposition. Postoperative mobility was also comparable to arthroscopic distal scaphoid resection (postoperative flexion-extension

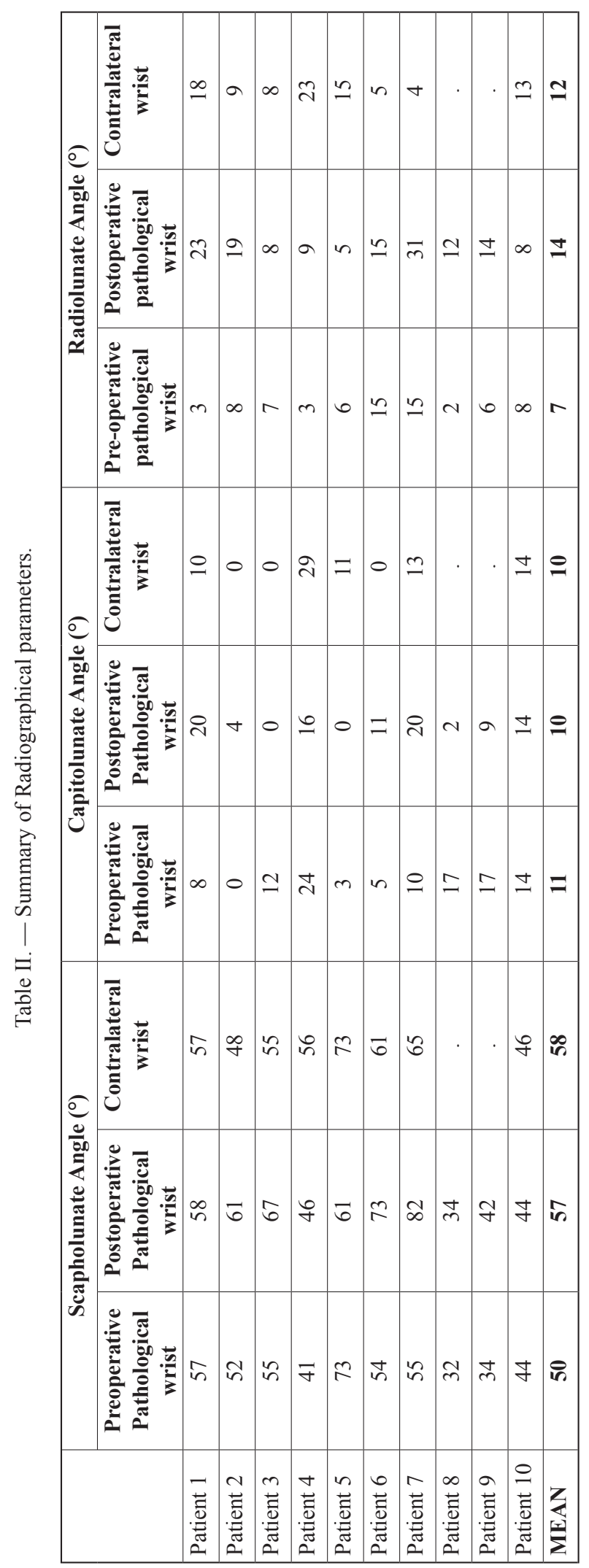


arc range $\left.115^{\circ}-143^{\circ}\right)(17,18)$ and distal scaphoid resection plus pyrocarbon implant (post-operative flexion-extension arc of $126^{\circ}$ ) (19). Postoperative range of motion after STT arthrodesis and partial trapeziectomy was more limited : flexion-extension arc ranged from $77^{\circ}$ to $103^{\circ}(20,21)$, respectively $100^{\circ}-108^{\circ}(22,23)$.

The current study showed that postoperative grip strength significantly increased after surgery ( $28 \mathrm{~kg}-95 \%$ of the contralateral side). This was better than previously measured by Garcia-Elias ( $84 \%$ of contralateral wrist) (5) and Berkhout (23kg) (16). Results for grip strength were comparable for distal scaphoid resection plus pyrocarbon implant (23-24 kg, 82\% versus contralateral wrist) $(24,25)$, partial trapeziectomy $(19 \mathrm{~kg}-24 \mathrm{~kg}, 100 \%$ versus contralateral) $(22,26)$ and arthroscopic distal scaphoid resection $(19 \mathrm{~kg}-42 \mathrm{~kg}, 79-100 \%)(4,18,27)$. Strength was more restricted after STT arthrodesis (range from $68 \%$ to $77 \%$ versus contralateral wrist) $(17,20,21,28)$ and wrist denervation $(69 \%$ versus contralateral wrist) (29). Postoperative VAS scores were low for all treatment modalities (range 0.6-3) $(15,16,18,19,21,22,25-27,30)$. Best functional results, with lowest DASH scores, were reported in the current study.

In the current study a mild postoperative increase in DISI deformity was seen, with three out of 10 wrists had postoperatively a RL angle $>15^{\circ}, 5$ out of ten wrist a SL angle $>60^{\circ}$ and 3 out of ten wrists a CL angle $>15^{\circ}$. This supports the prior findings that excisional arthroplasty might worsen carpal instability (30). Several studies were conducted to evaluate the effect of carpal instability on functional outcome, but neither the amount of DISI (15) nor the resection height were significantly correlated with the functional scores $(5,15,31)$. This was confirmed by the current study in which no correlation between amount of resected distal scaphoid or radiographic parameters and Q-DASH or PRWHE scores was found.

Postoperative pain was minimal with average VAS score of 1.75 and the maximum VAS score was 3.0. This is in line with Berkhout and Macuzzi after distal scaphoid resection who reported an average pain score of 2.1 on a 0 to 10 scale $(16,30)$.

Advantage of the present study is the long time to follow-up. This is longer than similar clinical studies on resection of distal pole of scaphoid for STT osteoarthritis $(5,16)$. This is the first study to present the results concerning the use of allograft tendon interposition after distal scaphoid resection for isolated STT arthritis. Disadvantage is the limited study group of 10 operated wrists. Because STT arthtitis is mostly asymptomatic and symptomatic STT arthritis tends to respond well to conservative treatment, surgery is restricted to a small amount of patients. Some pre-operative measures were missing because they were not noted in the patient files. We did not have any pre-operative measures of Q-DASH, PRWHE and VAS scores, so postoperative changes in these parameters could not be measured.

\section{CONCLUSION}

The current study indicates that distal scaphoid resection for isolated STT arthritis is a save procedure with minimal complications. It significantly improves grip strength after surgery. Mobility of the wrist was well preserved after surgery and similar to contralateral wrist. Pain postoperative was very limited (low VAS scores) and good functional scores (Q-DASH and PRWHE) were noted. Our findings support prior findings that excisional arthroplasty might worsen carpal instability.

\section{REFERENCES}

1. Wollstein R, Clavijo J, Gilula LA. Osteoarthritis of the Wrist STT Joint and Radiocarpal Joint. Arthritis. 2012 ; $2012: 1-5$.

2. Kapoutsis D V., Dardas A, Day CS. Carpometacarpal and scaphotrapeziotrapezoid arthritis: Arthroscopy, arthroplasty, and arthrodesis. J Hand Surg Am [Internet]. 2011; 36(2): 354-66. Available from: http://dx.doi. org/10.1016/j.jhsa.2010.11.047

3. Wu JC, Calandruccio JH. Evaluation and Management of Scaphoid-Trapezium-Trapezoid Joint Arthritis. Orthop Clin North Am [Internet]. 2019 ; 50(4) : 497-508. Available from : https://doi.org/10.1016/j.ocl.2019.05.005

4. Iida A, Omokawa S, Kawamura K, Shimizu T, Onishi T, Tanaka Y. Arthroscopic Distal Scaphoid Resection for Isolated Scaphotrapeziotrapezoid Osteoarthritis. J Hand Surg Am [Internet]. 2019 ; 44(4) : 337.e1-337.e5. Available from : https://doi.org/10.1016/j.jhsa.2018.06.021 
5. Garcia-Elias M. Excisional arthroplasty for scaphotrapeziotrapezoidal osteoarthritis. $J$ Hand Surg Am [Internet]. $2011 ; 36(3)$ : 516-20. Available from : http:// dx.doi.org/10.1016/j.jhsa.2010.12.016

6. Deans VM, Naqui Z, Muir LTSW. Scaphotrapeziotrapezoidal Joint Osteoarthritis : A Systematic Review of Surgical Treatment. J hand Surg AsianPacific Vol. 2017 ; 22(1) : 1-9.

7. Merel J.-L. Berkhout, Qiqi Yin MJPFR. Current Trends in Operative Treatment of Scaphotrapeziotrapezoid Osteoarthritis : A Survey among European Hand Surgeons. J Wrist Surg. 2020 ; 9 : 94-99.

8. Corbin C, Warwick D. Midcarpal instability after excision arthroplasty for scapho-trapezial-trapezoid (STT) arthritis. J Hand Surg Eur Vol. 2009 ; 34(4) : 537-8.

9. Kamal RN, Chehata A, Rainbow MJ, Llusá M, GarciaElias M. The effect of the dorsal intercarpal ligament on lunate extension after distal scaphoid excision. J Hand Surg Am [Internet]. $2012 ; 37(11): 2240-5$. Available from : http://dx.doi.org/10.1016/j.jhsa.2012.07.029

10. Beaton DE, Wright JG, Katz JN, Amadio P, Bombardier C, Cole D, et al. Development of the QuickDASH : COmparison of three item-reduction approaches. $J$ Bone $J t$ Surg - Ser A. $2005 ; 87(5)$ : 1038-46.

11. MacDermid JC. Development of a scale for patient rating of wrist pain and disability. J Hand Ther [Internet]. 1996 ; 9(2) : 178-83. Available from : http://dx.doi.org/10.1016/ S0894-1130(96)80076-7

12. A. K. Clinical test of apposition and counter-apposition of the thumb. Ann Chir Main. 1986 ; 5(1) : 67e73. Ann Chir Main. $1986 ; 5(1): 67-73$.

13. Tan S, Ghumman SS, Ladouceur M, Moser TP. Carpal angles as measured on CT and MRI: can we simply translate radiographic measurements? Skeletal Radiol. 2014 ; 43(12) : 1721-8.

14. White L, Clavijo J, Gilula LA, Wollstein R. Classification system for isolated arthritis of the scaphotrapeziotrapezoidal joint. J Plast Surg Hand Surg. 2010 ; 44(2) : 112-7.

15. Garcia-Elias M, Lluch AL, Farreres A, Castillo F, Saffar P. Resection of the distal scaphoid for scaphotrapeziotrapezoid osteoarthritis. J Hand Surg Am. 1999 ; 24 B(4) : 448-52.

16. Berkhout MJL, Bachour Y, Wessing D, Ritt MJPF. Distal Pole Resection of the Scaphoid for the Treatment of Scaphotrapeziotrapezoid Osteoarthritis. Hand [Internet]. 2019 ; 14(2): 230-5. Available from: https://doi.org/ $10.1177 / 1558944717735939$

17. Frykman EB, Af Ekenstam F, Wadin K. Triscaphoid arthrodesis and its complications. J Hand Surg Am. 1988 ; 13(6) : 844-9.

18. Ashwood N, Bain GI, Fogg Q. Results of arthroscopic debridement for isolated scaphotrapeziotrapezoid arthritis. J Hand Surg Am. 2003 ; 28(5) : 729-32.
19. Low AK, Edmunds IA. Isolated scaphotrapeziotrapezoid osteoarthritis : preliminary results of treatment using a pyrocarbon implant. Hand Surg. 2007 ; 12(2) : 73-7.

20. Watson HK, Ballet FL. The SLAC wrist: Scapholunate advanced collapse pattern of degenerative arthritis. $J$ Hand Surg Am [Internet]. 1984 ; 9(3) : 358-65. Available from : http://dx.doi.org/10.1016/S0363-5023(84)80223-3

21. Minami A, Kato H, Suenaga N, Iwasaki N. Scaphotrapeziotrapezoid fusion: Long-term follow-up study. $J$ Orthop Sci. 2003 ; 8(3) : 319-22.

22. Andrachuk J, Yang SS. Modified total trapezial and partial trapezoidal excision and ligament reconstruction tendon interposition reduces symptoms in isolated scaphotrapezialtrapezoid arthritis of the wrist. J Hand Surg Eur Vol. 2012 ; 37(7) : 637-41.

23. Langenhan R, Hohendorff B PA. Trapeziectomy and ligament reconstruction tendon interposition for isolated scaphotrapeziotrapezoid osteoarthritis of the wrist. J Hand Surg Eur. 2014 ; Vol. 2014(Oct ; 39(8)) : 833-7.

24. Kansagra SM, Cohen MK. The Opioid Epidemic in NC : Progress, Challenges, and Opportunities. N C Med J. 2018 ; 79(3) : 157-62.

25. Pegoli L, Pozzi A, Pivato G, Luchetti R. Arthroscopic Resection of Distal Pole of the Scaphoid for Scaphotrapeziotrapezoid Joint Arthritis : Comparison between Simple Resection and Implant Interposition. J Wrist Surg. 2016 ; 05(03) : 227-32.

26. Langenhan R, Hohendorff B, Probst A. Trapeziectomy and ligament reconstruction tendon interposition for isolated scaphotrapeziotrapezoid osteoarthritis of the wrist. J Hand Surg Eur Vol. 2014 ; 39(8) : 833-7.

27. Normand J, Desmoineaux $\mathbf{P}$, Boisrenoult $\mathbf{P}$, Beaufils P. Résection arthroscopique du pôle distal du scaphoïde dans l'arthrose scapho-trapézo-trapézoidienne. Chir Main [Internet]. 2012 ; 31(1) : 13-7. Available from: http:// dx.doi.org/10.1016/j.main.2011.11.005

28. Fortin PT, Louis DS. Long-term follow-up of scaphoidtrapezium-trapezoid arthrodesis. J Hand Surg Am. 1993 ; 18(4) : 675-81.

29. Braga-Silva J, Romn JA, Padoin A V. Wrist denervation for painful conditions of the wrist. J Hand Surg Am [Internet]. 2011 ; 36(6) : 961-6. Available from : http:// dx.doi.org/10.1016/j.jhsa.2011.03.004

30. Marcuzzi A, O"zben H, Russomando A. Treatment of scaphotrapezial trapezoidal osteoarthritis with resection of the distal pole of the scaphoid. Acta Orthop Traumatol Turc. 2014 ; 48(4) : 431-6.

31. Brouwers D, Decramer A, Cornu P, Le Viet D. Does the DISI matter after distal scaphoidectomy with tendon interposition for STT osteoarthritis? Hand Surg Rehabil [Internet]. 2020 ; 39(4) : 284-90. Available from : https:// doi.org/10.1016/j.hansur.2020.03.005 